\title{
An Analysis of the Relationship between Grit and the Psychological Well-Being of Psychiatry Residents
}

\author{
Dain Kim, MD', Seung-Ho Jang, MD², Sung-Yong Park, MD \\ ${ }^{1}$ Department of Psychiatry, Keyo Hospital, Uiwang, ${ }^{2}$ Department of psychiatry, Wonkwang University Hospital, Iksan, Korea
}

Background: Grit is a psychological factor that is defined as "perseverance and passion for the long term." A growing interest exists in this factor because it aids in overcoming difficult tasks related to the psychological well-being of psychiatry residents to prevent burnout; however, it is still under-recognized in the Korean clinical practices. This study therefore examined the relationship between grit and the psychological well-being of psychiatry residents.

Methods: In all, 77 psychiatry residents completed the study survey, and all met our study's participation criteria. To assess these residents' grit, burnout, and psychological well-being, the Original Grit Scale, Maslach Burnout Inventory, and Psychological Well-being Scale were employed. For the statistical comparison, t-test, ANOVA, and Pearson correlation analysis were used.

Results: A significant relationship prevailed between grit and psychological well-being. The grit scores had positive and negative correlations with psychological well-being $(p<0.01)$ and burnout $(p<0.01)$, respectively. Thus, psychiatry residents with higher grit scores were more likely to experience less burnout and have higher psychological well-being scores.

Conclusion: A significant relationship existed between grit, psychological well-being, and burnout. Grit assessment thus aided in identifying psychiatry residents who were at a greater risk of quitting their training program due to poor psychological well-being or emotional exhaustion.

Keywords Grit; Mental health; Residency; Burnout

\section{INTRODUCTION}

Resident or resident's training is specifically a stage of graduate medical education. It refers to a qualified physician who practices medicine, usually in a hospital or clinic, under the direct or indirect supervision of a senior medical clinician registered in that specialty such as an attending physician or consultant. The resident's training process is a key process for fostering professional medical personnel, and residents experience a great deal of stress during this period. In a survey of American residents, residents rated most work environments 4 or 5 on the five-point Likert scale (very stressful) [1]. Although laws are being implemented in Korea to better the training environment and status of residents in order to improve their excessive training period and poor training environment, they are still experiencing much stress at their jobs. Residents frequently complained of physiological imbalance and chronic fatigue due to the burden from patients, long working hours and irregular sleep hours. As a result, they were found to feel anxious about not being able to perform their duties safely. Excessive job stress and reduced attention are likely to lead to medical errors and psychological burnout, and residents with such experiences may show reduced job satisfaction and organizational immersion, leading to poor medical quality or discontinuation of the training process.

Received October 31, 2020, Revised November 3, 2020, Accepted November 4, 2020

Correspondence: Sung Yong Park, MD

Department of Psychiatry, Keyo Hospital, 15 Ojeon-ro, Uiwang 16062, Korea

TEL +82-31-455-3333 FAX +82-31-452-4110 E-mail woeorl@daum.net ORCID https://orcid.org/0000-0002-8685-620X

Copyright $@$ by Korean Society for Affective Disorders. All Rights reserved.

This is an Open Access article distributed under the terms of the Creative Commons Attribution Non-Commercial License (http://creativecommons.org/licenses/ by-nc/4.0/) which permits unrestricted non-commercial use, distribution, and reproduction in any medium, provided the original work is properly cited. 
Recently, various studies have been conducted overseas on residents' job satisfaction, psychological wellbeing, and burnout, but not many studies have been conducted on residents' job satisfaction or psychological well-being in Korea. Past studies have emphasized external motivations such as leadership, communication style, organizational culture, and welfare as predisposing factors affecting organizational immersion, but recent studies have highlighted the importance of personal competency [2], but there is no accurate understanding of the factors that affect personal competence and psychological well-being that enable the resident to endure difficult times or situations.

Therefore this study focused on grit, which is one of the characteristics of personal competence. Grit defines passion and perseverance for long-term goals as key factors in predicting success and achievement, which is the power to accomplish the goals you are aiming to achieve and the ability to consistently work toward them for a long time, even if there are difficulties, adversity and slumps [3,4]. Duckworth [3] found the concept of grit in six studies of different population groups. Therefore this study focused on grit, which is one of the characteristics of personal competence. Grit defines passion and perseverance for long-term goals as key factors in predicting success and achievement, which is the power to accomplish the goals you are aiming to achieve and the ability to consistently work toward them for a long time, even if there are difficulties, adversity and slumps $[3,4]$. Duckworth [3] found the concept of grit in six studies of different population groups. In a West Point study of those who gave up in the middle of the six-week training before school entrance and those who made it to the end, as well as in a study of Ivy League students, Duckworth [3] reported that grit, rather than intelligence or talent, predicts achievement in various areas targeted by individuals. In short, person with higher Grit seems to tolerate more and likely to complete difficult tasks.

Also, studies show that the higher one's psychological well-being, the more capable one is of controlling one's behavior independently or on one's own, and the more motivated one is to realize one's potential [5]. As the degree of happiness or satisfaction with a person's overall life, psychological well-being is defined as the subjective sense of happiness or satisfaction with one's overall life $[6,7]$. In other words, studies show that people with a better sense of happiness or a higher sense of psychological well-being graduate by completing the university course, are less likely to quit their jobs, and are less likely to experience psychological burnout [8].

In December 2018, a patient receiving treatment murdered the psychiatrist with a weapon, raising social interest and awareness about the risk of violence in mental patients, as well as increasing social demand for mental health [9]. In order to protect psychiatrists from such violence, the so-called 'Im Se-won Act' has been pushed forward with the revision of the Mental Health Welfare Act, but measures for medical staff's mental health are still insufficient [10]. In psychiatry, violent behavior by patients is understood as a psychopathology. Relationship conflicts in special environments, such as the role of doctors amidst rapid social changes, confidentiality and high ethical awareness that are more emphasized than in other departments, and high work tension that must be prepared for in the event of a psychiatric emergency such as patients' suicide or violence, are found to cause stress [11]. Due to this special nature of psychiatry, residents who train in the department of psychiatry experience more stress of a special nature than those in other departments.

Therefore, in this study we wanted to find out the correlation between grit and psychological well-being in psychiatry residents and explore it so that it could be used as basis material for psychological burnout and low psychological well-being that can be further mediated.

\section{MATERIALS AND METHODS}

\section{Participants}

This study was conducted on psychiatry residents who currently work at domestic training hospitals. Similar studies on the relation between burnout and psychological well-being in foreign residents studied 100 to 200 residents in various fields, but there has been no study on psychiatry residents alone. With those who expressed consent to participate in this study, their demographic and clinical data were collected and evaluated through a self-reporting questionnaire. The demographic data examined gender, age, marital status, child status, educational level, religion, socioeconomic level and training hospital environment, and clinical data evaluated grit, burnout and psychological well-being.

This study has been approved by the Clinical Ethics Committee of Wonkwang University Medical Center 
(WKUH 2020-05-049-001), the selection criteria were psychiatry residents undergoing training who agreed to participate in this study and who can read and understand the questionnaire, and those who disagreed to participate in the study and those who did not respond were excluded. From the IRB approval date to December 31, 2020 , the samples were specified as psychiatry residents aiming for at least 50 people, and a final 77 were selected.

\section{Assessment}

\section{1) Grit: Original Grit Scale}

The Original Grit Scale (Grit-O), which was developed to measure grit, was adapted for domestic conditions and a validated scale was used [12]. There are 12 questions in total, consisting largely of two sub-regions: consistency of interest and perseverance of effort, with six questions for each. A higher score on the five-point Likert scale indicates higher grit.

\section{2) Burnout: Maslach Burnout Inventory}

A tool that modified and supplemented the Maslach Burnout Inventory (MBI) [13] was used for burnout. There are 22 questions in total, consisting of four main questions: eight for emotional burnout, eight for personal accomplishment, three for depersonalization, and three for interpersonal relationships. A higher score on the five-point Likert scale indicates a higher degree of burnout.

\section{3) Psychological Well-Being Scale}

For psychological well-being, an abbreviated version of the Psychological Well-Being Scale (PWS), adapted and validated for domestic purposes, was used [14]. A total of 46 questions were abbreviated to 18 questions, consisting largely of six sub-components. There were three questions for self-acceptance, three for positive interpersonal relationships, three for autonomy, three for purpose in life, three for environmental mastery, and three for personal growth. A higher score on the fivepoint Likert scale indicates a higher level of psychological well-being.

\section{Statistical analysis}

IBM SPSS Statistics for Windows, Version 22.0 (IBM Co., Armonk, NY, USA) was used to verify overall data normality, for correlation analysis, factor analysis and reliability analysis. To determine whether the sociodemographic characteristics of the subjects are related to psychological well-being, differences between groups of continuous variables were analyzed using t-test or

Table 1. Sociodemographic characteristics of participants

\begin{tabular}{|c|c|}
\hline Categorical variable & Value $(n=77)$ \\
\hline \multicolumn{2}{|l|}{ Gender $(n=77)$} \\
\hline Man & $41(53.2)$ \\
\hline Woman & $36(46.8)$ \\
\hline \multicolumn{2}{|l|}{ Age $(n=77)$} \\
\hline$>20 y$ & $21(27.3)$ \\
\hline$\geq 30 y$ & $55(71.4)$ \\
\hline$\geq 40 y$ & $1(1.3)$ \\
\hline \multicolumn{2}{|l|}{ Marital status $(n=77)$} \\
\hline Single & $52(67.5)$ \\
\hline Married & $25(32.5)$ \\
\hline \multicolumn{2}{|l|}{ Child Status $(n=25)$} \\
\hline Yes & $9(11.7)$ \\
\hline No & $16(20.8)$ \\
\hline \multicolumn{2}{|l|}{ Religion ( $n=77$ ) } \\
\hline Yes & $32(41.6)$ \\
\hline No & $45(58.4)$ \\
\hline \multicolumn{2}{|l|}{ Type of training hospital } \\
\hline University hospital & $54(70.1)$ \\
\hline Secondary general hospital & $7(9.1)$ \\
\hline Psychiatric hospital & $15(19.5)$ \\
\hline \multicolumn{2}{|c|}{ Table of organizations of residency } \\
\hline 1 person/year & $6(7.8)$ \\
\hline 2 person/year & $56(72.7)$ \\
\hline$\geq 3$ person/year & $15(19.5)$ \\
\hline \multicolumn{2}{|c|}{ Income (per year, thousand KRW) } \\
\hline$<2,600$ & $1(1.3)$ \\
\hline $2,600-3,000$ & $1(1.3)$ \\
\hline $3,000-3,400$ & $1(1.3)$ \\
\hline $3,400-3,800$ & $15(19.5)$ \\
\hline $3,800-4,200$ & $18(23.4)$ \\
\hline$\geq 4,200$ & $41(53.2)$ \\
\hline \multicolumn{2}{|l|}{ Job satisfaction as doctor } \\
\hline Very satisfied & $7(9.1)$ \\
\hline Satisfied & $39(50.6)$ \\
\hline So so & $28(36.4)$ \\
\hline Unsatisfied & $3(3.9)$ \\
\hline \multicolumn{2}{|c|}{ Satisfaction as psychiatry resident } \\
\hline Very satisfied & $13(16.9)$ \\
\hline Satisfied & $46(59.7)$ \\
\hline So so & $16(20.8)$ \\
\hline Unsatisfied & $2(2.6)$ \\
\hline
\end{tabular}

Values are presented as number (\%). 
one-way ANOVA. Pearson Correlation analysis was conducted for a correlation analysis between grit and variables that could affect psychological well-being and burnout, and the relationship between the lower scales of each scale and grit was also analyzed. In addition, a stepwise multiple regression analysis was conducted to find out the relative influence on residents' grit, psychological well-being and burnout. Because the sample size was rather small at 77, a modified $\mathrm{R}$ value was used, calculated by calibrating to degrees of freedom to prevent bias by random measurement error.

\section{RESULTS}

\section{Socio-demographic characteristics}

The socio-demographic characteristicss of the study subjects are shown in Table 1 . Out of a total of 77, by age they were: $20 \mathrm{~s}(27.3 \%), 30 \mathrm{~s}(71.4 \%)$, and $40 \mathrm{~s}(1.3 \%)$, with 41 men (53.2\%) and 36 women (46.8\%). Fifty-two (67.5\%) were unmarried, 22 (32.5\%) married; for child status $9(36 \%)$ said 'with children' and $16(64 \%)$ said 'no children'; for training environment, 54 (70.1\%) said university hospital, followed by seven $(9.1 \%)$ at secondary general hospital and $15(19.5 \%)$ at psychiatric hospital. For doctors' job satisfaction and satisfaction with choosing a major in psychiatry, most said 'satisfied' (50.6\%, $59.7 \%$ ) for each out of very satisfied, satisfied, okay, and dissatisfied. The average daily working hours of resi- dents differed to some extent depending on the training environment, with $10.42 \pm 2.08$ hours per day, $1.41 \pm 1.29$ days on duty per week, $5.19 \pm 1.22$ hours of sleep per week when on duty, and $6.91 \pm 0.96$ hours per day when not on duty. The average number of in-patients a resident was in charge of was 5.16 \pm 7.14 , with a very large deviation depending on the training hospital.

\section{Differences in clinical characteristics by socio-psychological factors}

\section{1) Relationship between grit, psychological well-being, and burnout}

A Pearson Correlation Analysis was performed to identify the relationship between grit, psychological well-being, and burnout. The results showed significant correlation in many cases (Table 2,3). Overall, there was a significant positive correlation between grit and psychological well-being, and a significant negative correlation between grit and burnout. In addition, an analysis of the correlation between the mean, standard deviation, kurtosis, and skewness of the sub-variables showed that the absolute value of skewness was less than 2 and the absolute value of kurtosis less than 7, which fulfilled the normality hypothesis, and thus subsequent analysis was conducted (Table 3). In particular, focusing on the lower scale of grit that we are interested in, consistency of interest had a significant positive correlation with purpose in life $(\mathrm{r}=0.382, \mathrm{p}<0.01)$, a sub-scale measure of psychological well-being, and the depersonalization $(r=0.330$,

Table 2. Correlation analysis of PWS, MBI, and 2 subscales of Grit-O

\begin{tabular}{|c|c|c|c|}
\hline & Consistency of interest & Perseverance of effort & Grit-0 \\
\hline \multicolumn{4}{|l|}{ PWS } \\
\hline Self-acceptance & -0.179 & $0.325 *$ & 0.158 \\
\hline Positive relationships & 0.049 & $0.264 \star \star$ & $0.247 * \star$ \\
\hline Autonomy & 0.152 & $0.300 *$ & $0.347 \star$ \\
\hline Purpose in Life & $-0.382 \star$ & $0.547 \star$ & $0.242 * \star$ \\
\hline Environmental mastery & -0.037 & $0.473 *$ & $0.410 \star$ \\
\hline Personal growth & -0.124 & $0.435 \star$ & $0.316 \star$ \\
\hline PWS total & -0.165 & $0.634 \star$ & $0.450 *$ \\
\hline \multicolumn{4}{|l|}{$\mathrm{MBI}$} \\
\hline Burnout & 0.177 & $-0.235 \star \star$ & -0.117 \\
\hline Depersonalization & $0.330 *$ & $-0.377 \star$ & -0.127 \\
\hline Personal accomplishment & 0.069 & $-0.583 \star$ & $-0.436 \star$ \\
\hline MBI total & $0.233 * \star$ & $-0.459 \star$ & $-0.260 \star \star$ \\
\hline
\end{tabular}

PWS, Psychological Well-being Scale; MBI, Maslach Burnout Inventory; Grit-0, Original Grit Scale.

$\star p<0.05, * \star p<0.01$. 


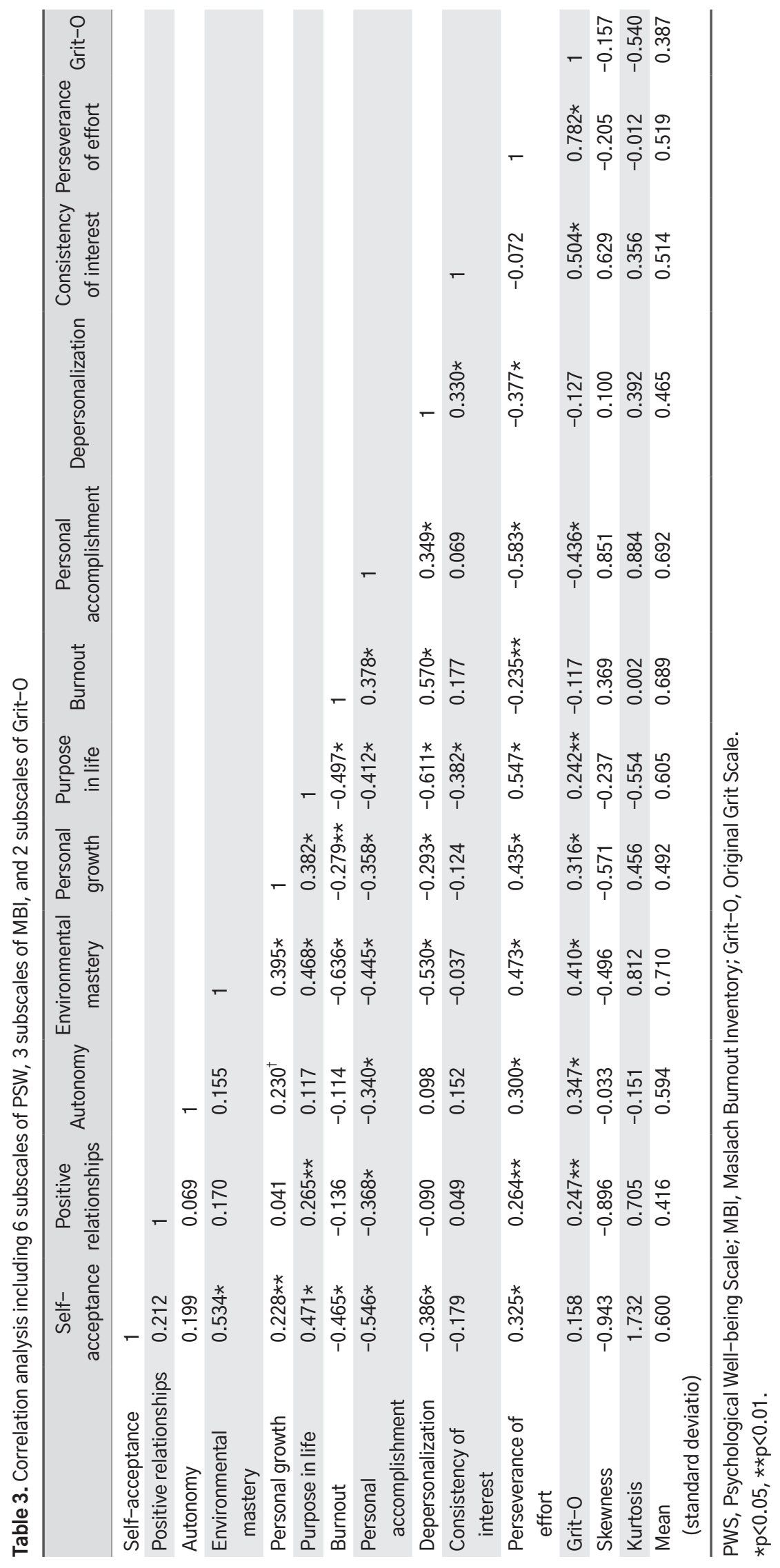


Dain Kim, et al. Mental Health of Psychiatry Residents

Table 4. The mediation effect of Grit on psychological well-being and burnout

\begin{tabular}{|c|c|c|c|c|c|c|c|c|}
\hline Dependent variable & Independent variable & B & SE & $\beta$ & $\mathrm{t}$ & $\mathrm{R}^{2}$ & $\operatorname{adj} R^{2}$ & $\mathrm{~F}$ \\
\hline \multicolumn{9}{|l|}{ PSW } \\
\hline \multirow[t]{3}{*}{ Self-acceptance } & (constant) & 2.839 & 0.585 & & 4.854 & 0.130 & 0.106 & 5.448 \\
\hline & Consistency of interest & -0.183 & 0.128 & -0.156 & -1.427 & & & \\
\hline & Perseverance of effort & 0.360 & 0.126 & 0.314 & 2.866 & & & \\
\hline \multirow[t]{3}{*}{ Positive relationships } & (constant) & 2.250 & 0.418 & & 5.387 & 0.074 & 0.049 & 2.962 \\
\hline & Consistency of interest & 0.055 & 0.091 & 0.068 & 0.609 & & & \\
\hline & Perseverance of effort & 0.215 & 0.090 & 0.269 & 2.394 & & & \\
\hline \multirow[t]{3}{*}{ Autonomy } & (constant) & 1.152 & 0.581 & & 1.983 & 0.120 & 0.096 & 5.058 \\
\hline & Consistency of interest & 0.201 & 0.126 & 0.174 & 1.592 & & & \\
\hline & Perseverance of effort & 0.358 & 0.125 & 0.313 & 2.861 & & & \\
\hline \multirow[t]{3}{*}{ Purpose in life } & (constant) & 2.444 & 0.565 & & 4.326 & 0.418 & 0.402 & $26.534 \star$ \\
\hline & Consistency of interest & -0.476 & 0.123 & -0.345 & -3.875 & & & \\
\hline & Perseverance of effort & 0.714 & 0.122 & 0.522 & 5.873 & & & \\
\hline \multirow[t]{3}{*}{ Environmental mastery } & (constant) & 2.072 & 0.452 & & 4.581 & 0.223 & 0.202 & $10.642 \star$ \\
\hline & Consistency of interest & -0.003 & 0.098 & -0.003 & -0.026 & & & \\
\hline & Perseverance of effort & 0.448 & 0.097 & 0.472 & 4.599 & & & \\
\hline \multirow[t]{3}{*}{ Personal growth } & (constant) & 2.510 & 0.565 & & 4.442 & 0.198 & 0.176 & $9.111 *$ \\
\hline & Consistency of interest & -0.110 & 0.123 & -0.093 & -0.892 & & & \\
\hline & Perseverance of effort & 0.498 & 0.122 & 0.428 & 4.099 & & & \\
\hline \multirow[t]{3}{*}{ Total PSW } & (constant) & 2.206 & 0.286 & & 7.724 & 0.417 & 0.401 & $26.444 \star$ \\
\hline & Consistency of interest & -0.084 & 0.062 & -0.120 & -1.348 & & & \\
\hline & Perseverance of effort & 0.432 & 0.061 & 0.626 & 7.030 & & & \\
\hline \multicolumn{9}{|l|}{$\mathrm{MBI}$} \\
\hline \multirow[t]{3}{*}{ Burnout } & (constant) & 2.972 & 0.689 & & 4.317 & 0.081 & 0.056 & 3.257 \\
\hline & Consistency of interest & 0.216 & 0.150 & 0.161 & 1.443 & & & \\
\hline & Perseverance of effort & -0.296 & 0.148 & -0.223 & -1.995 & & & \\
\hline \multirow[t]{3}{*}{ Depersonalization } & (constant) & 2.705 & 0.631 & & 4.285 & 0.234 & 0.213 & $11.305 *$ \\
\hline & Consistency of interest & 0.410 & 0.137 & 0.304 & 2.983 & & & \\
\hline & Perseverance of effort & -0.473 & 0.136 & -0.355 & -3.478 & & & \\
\hline \multirow[t]{3}{*}{ Personal accomplishment } & (constant) & 4.375 & 0.394 & & 11.107 & 0.341 & 0.323 & $19.135 *$ \\
\hline & Consistency of interest & 0.025 & 0.086 & 0.027 & 0.286 & & & \\
\hline & Perseverance of effort & -0.521 & 0.085 & -0.581 & -6.143 & & & \\
\hline \multirow[t]{3}{*}{ Total burnout } & (constant) & 3.421 & 0.440 & & 7.775 & 0.251 & 0.231 & $12.402 *$ \\
\hline & Consistency of interest & 0.191 & 0.096 & 0.201 & 1.990 & & & \\
\hline & Perseverance of effort & -0.418 & 0.095 & -0.445 & -4.410 & & & \\
\hline
\end{tabular}

SE, standard error; PWS, Psychological Well-being Scale; MBI, Maslach Burnout Inventory. *p<0.01.

$\mathrm{p}<0.01$ ), a sub-scale measure of burnout.

On the other hand, perseverance of effort showed a significant positive correlation with the following submeasures of psychological well-being: self-acceptance $(\mathrm{r}=0.325, \mathrm{p}<0.01)$, positive interpersonal relationship $(\mathrm{r}=0.264, \mathrm{p}<0.05)$, autonomy $(\mathrm{r}=0.300, \mathrm{p}<0.01)$, environmental mastery $(\mathrm{r}=0.473, \mathrm{p}<0.05)$, personal growth $(\mathrm{r}=0.435, \mathrm{p}<0.01)$, and purpose in life $(\mathrm{r}=0.547, \mathrm{p}<0.01)$. Perseverance of effort also showed significant results in emotional burnout, personal accomplishment, and de- personalization, which are lower factors of the burnout scale. There was a significant negative correlation with emotional burnout $(\mathrm{r}=-0.235, \mathrm{p}<0.05)$, personal accomplishment $(\mathrm{r}=-0.583, \mathrm{p}<0.01)$, and depersonalization $(\mathrm{r}=-0.377, \mathrm{p}<0.01)$.

\section{2) Influence on the relationship between psychiatry residents' grit, psychological well-being, and burnout}

First, psychiatry residents' grit explains $41.7 \%$ of total 
psychological well-being, which was statistically significant $(\mathrm{F}=26.44, \mathrm{p}<0.01)$. There was a significant negative correlation between overall psychological well-being of psychiatry residents and consistency of interest $(\beta=-0.12)$ and a significant positive correlation with perseverance of effort ( $\beta=0.626$ ) (Table 4).

By sub-region, psychiatry residents' grit did not have a significant impact on self-acceptance, positive interpersonal relationship, and autonomy, but had a $41.8 \%$ influence on purpose in life, $22.3 \%$ influence on environmental mastery, and $19.8 \%$ influence on personal growth, which was statistically significant. In the case of purpose in life, which is explained at the highest rate, there was a significant positive correlation between grit and consistency of interest $(\beta=0.068)$ and grit and perseverance of effort (0.269). In terms of environmental mastery, the influence of grit on consistency of interest was $(\beta=-0.003)$ and on perseverance of effort $(\beta=0.472)$, and in personal growth consistency of interest was $(\beta=-0.892)$ and perseverance of effort $(\beta=0.428)$, showing a negative and positive correlation, respectively.

Second, psychiatry residents' grit explains $25 \%$ of overall burnout, which is also statistically significant $(\mathrm{F}=12.402, \mathrm{p}<0.01)$. The overall burnout of residents and consistency of interest $(\beta=0.201)$ had a negative correlation, while with perseverance of effort it was $(\beta=-0.445)$.

By sub-region, it was shown that grit had a significant impact on depersonalization and personal accomplishment, excepting emotional burnout. Residents' grit explained depersonalization statistically significantly at $23.4 \%$ and personal accomplishment at $34.1 \%$. In the case of depersonalization, grit had a positive correlation with consistency of interest $(\beta=0.304)$ and a negative one with perseverance of effort $(\beta=-0.355)$, and in the case of personal accomplishment grit had a positive correlation with consistency of interest $(\beta=0.027)$ and a negative correlation with perseverance of effort $(\beta=-0.355)$. Based on these results, the effect of residents' grit on their psychological well-being and burnout is significant.

\section{DISCUSSION}

This study was conducted on domestic psychiatry residents to study the influence of grit, which is a personality trait of residents, on psychological well-being and burnout. An analysis of the relationship between residents' grit, psychological well-being, and burnout showed a significant correlation. Consistent with the hypothesis of the study, residents' grit showed a positive correlation with psychological well-being and a negative correlation with psychological burnout. These results are consistent with previous studies, and a higher measurement level of grit indicated lower the stress and psychological burnout levels [15]. In other words, a doctor with a high level of grit is less likely to experience burnout, and a doctor with a high level grit may experience a high level of psychological well-being.

The influence of grit was high in the following order: purpose in life, personal accomplishment, environmental mastery, depersonalization, and personal growth. Grit had an especially high level of influence in purpose in life, personal accomplishment, and environmental. This indicates that grit has a positive influence on purpose in life and personal accomplishment, and suggests that it will serve as an important psychological factor for residents who are under a lot of job stress and have a long training period. In this study, $76.6 \%$ of residents answered 'high' in regard to whether they were satisfied with their choice of major. Those who answered 'high' had a lower score for psychological burnout than those who answered otherwise, which is explained as a result of prior studies that showed that grit has an impact on self-esteem and satisfaction in life.

In addition, an analysis of the correlation between perseverance of effort and consistency of interest, which are sub-factors of grit, and the lower scales of psychological well-being and burnout showed that perseverance of effort has a significant positive correlation with all the lower scales of psychological well-being and a significant negative correlation with all the sub-factors of psychological burnout. However, the same correlation was not shown for the above sub-scales in the case of consistency of interest, which is likely related to the small sample group, which is the limitation of this study.

The results of this study showed that measuring the grit of residents can be a predictor of their overall psychological well-being or burnout. In the U.S., where most of the studies on the grit, which is a non-cognitive feature of individuals, are conducted, there are many studies $[16,17]$ conducted on residents in surgery or emergency departments. A study measuring grit among about 400 orthopedic residents reported that the grit score of most residents was higher than that of the general sample [18] and that the grit score was an independent 
factor predicting psychological burnout or psychological well-being. A study of 139 residents in internal medicine also found that low grit scores and high psychological measurements made it possible to predict psychological burnout, psychological well-being and depression [19].

But so far, there have been studies only on programs that reduce psychological well-being and burnout in residents rather than on programs that objectively measure and promote grit. In one study, residents voluntarily participated in a 12 -week curriculum in which a 60 -minute lecture and a subsequent 15-minute booster lecture were conducted, but there were not enough results due to surgery residents' lack of time. There is a study that shows that implementing the Stress Management and Resilience Training program for U.S. radiology medical staff reduced psychological burnout and stress with a single medical intervention of just 90 minutes [20]. If the above program is adapted for the domestic situation along with the development of an objective evaluation tool for grit, it will not be difficult for residents with limited time to participate.

The limitations of this study are as follows. First, this study was conducted on psychiatry residents and the samples was small. It is possible that the statistics were not significant due to the small sample. Second, this study identified three correlations of grit, burnout, and psychological well-being, but it was difficult to grasp their causal relationship. Grit may have had an effect on burnout and psychological well-being, but it may have been affected by them too, and there were no significant results for various demographic factors that would affect burnout and psychological well-being, such as marital status, income level, or child status. In the future, analytical studies will be needed to make forwardlooking research suggestions or to infer the causality. Third, although the survey was conducted by dividing the various training environments, such as psychiatric hospitals, secondary general hospitals, and university hospitals, there are limitations in considering the impact of these different working environments and organizational cultures, and there is a limit to generalizing for all residents. However, this study is meaningful in that it is a full-fledged study of psychiatry residents.

Fourth, since it was a one-dimensional, cross-sectional study, there is something to be desired in identifying the relation between the progress of changes in residents' grit, burnout, and psychological well-being. According to Duckworth's study [4], there are studies that report that grit increases as age increases, which suggests that grit may change as years of experience increase. In other words, we suggest that it would be clinically meaningful to study measuring grit over a long period of time in more samples of residents by supplementing these limitations. Finally, it is possible that the subjects underestimated and overestimated due to the self-report bias because all the variables used consisted of self-reporting questionnaires, due to the limitations of this study. Objective observational research will be needed, such as using various research methods and perspectives, including observation and interview. Also, more research is needed on the non-cognitive nature of grit.

Despite these limitations, grit is a principal factor that affects achievement in early childhood education, academic achievement, and other various fields [21] and has shown results consistent with numerous prior research results on the association of residents' grit measurement and burnout. In other words, it is a study that showed that grit measurement of residents is clinically useful in identifying their psychological well-being and burnout, and it is meaningful that it can provide basic material for future research. Based on the results of this study, the need for developing objective tools and programs for the measurement of residents' grit, and the development of these tools will be of great clinical benefit to the selection and identification of individuals who are likely to give up training due to burnout and low job satisfaction due to their high stress level, depression and anxiety.

\section{CONCLUSION}

This study was consistent with the results of a number of prior studies in which a high level of grit in individuals indicates less psychological burnout. Also, by looking at personal internal factors such as grit and the effects on burnout and psychological well-being which are affected by environmental factors, it could be the basis for supporting education and counselling programs to enhance individual grit of residents in improving the hospital training environment. In developing programs to improve the training environment of residents, individual grit measurements will also help select individuals who are likely to give up during training due to burnout resulting from high stress, low job satisfaction, depression or anxiety. The PSW or MBI used as an assessment scale of the above training environment may 
be used immediately, but since the above measures include rather sensitive questions, respondents may not answer them correctly due to the bias of trying to answer them well, and since the Grit-O scale consists of short and less sensitive questions, it was confirmed that it was a suitable tool for clinical evaluation. In conclusion, measuring and evaluating residents' grit is of great clinical significance and will be of great help in improving their training environment.

\section{CONFLICTS OF INTEREST}

The authors have nothing to disclose.

\section{ORCID}

\author{
Dain Kim \\ https://orcid.org/0000-0003-1645-3154 \\ Seung-Ho Jang \\ https://orcid.org/0000-0002-3479-0552 \\ Sung-Yong Park \\ https://orcid.org/0000-0002-8685-620X
}

\section{REFERENCES}

1. Cohen JS, Patten S. Well-being in residency training: a survey examining resident physician satisfaction both within and outside of residency training and mental health in Alberta. BMC Med Educ 2005;5:21.

2. Levin HM. More than just test scores. Prospects 2012;42: 269-84.

3. Duckworth AL. Intelligence is not enough: Non -IQ predictors of achievement [PhD dissertation]. Philadelphia: University of Pennsylvania; 2006.

4. Duckworth AL, Peterson C, Matthews MD, Kelly DR. Grit: perseverance and passion for long-term goals. J Pers Soc Psychol 2007;92:1087-101.

5. Kim MS, Kim HY, Cha KH. Analyses on the construct of psychological well-being (PWB) of Korean male and female adults. Korean J Soc Personal Psychol 2001;15:19-39.

6. Andrews FM, Robinson JP. Measures of subjective wellbeing. In Robinson JP, Shaver PR, Wrightsman LS, eds. Measures of personality and social psychological attitudes. San Diego: Academic Press; 1991. p. 61-114.
7. Diener E. Subjective well-being. Psychol Bull 1984;95:54275.

8. Lyubomirsky S, King L, Diener E. The benefits of frequent positive affect: does happiness lead to success? Psychol Bull 2005;131:803-55.

9. Prince M, Patel V, Saxena S, Maj M, Maselko J, Phillips MR, et al. No health without mental health. Lancet 2007; 370:859-77.

10. Kim Sunup. Newspim.com [Internet]. Seoul: Newspim Communication [updated 2019 Jan 9; cited 2019 Jan 9]. Available from: http://www.newspim.com/news/ view/20190109000250.

11. Lee HJ, Kim JY. Effects of psychiatric nurses' secondary traumatic stress and compassion satisfaction on burnout: the moderating effect of social support. J Korean Acad Psychiatr Ment Health Nurs 2016;25:399-408.

12. Lee SR. Effects of grit, deliberate practice and contingencies of self-worth on academic achievement [PhD dissertation]. Seoul: Yonsei University; 2015.

13. Maslach C, Jackson SE. The Maslach burnout inventoryhuman services survey: MBI-HSS. In: Maslach C, Jackson SE, Leiter MP, eds. Maslach burnout inventory: manual. Menlo Park: Mind Garden; 2016.

14. Ryff CD. Happiness is everything, or is it? Explorations on the meaning of psychological well-being. J Personal Soc Psychol 1989;57:1069-81.

15. Lee SB, Bae EH, Sohn YW, Lee SR. Grit as a buffer against negative feedback: the effect of grit on emotional responses to negative feedback. Korean J Soc Personal Psychol 2016; 30:25-45.

16. Dam A, Perera T, Jones M, Haughy M, Gaeta T. The relationship between grit, burnout, and well-being in emergency medicine residents. AEM Educ Train 2018;3:14-9.

17. Salles A, Cohen GL, Mueller CM. The relationship between grit and resident well-being. Am J Surg 2014;207:251-4.

18. Kurian EB, Desai VS, Turner NS, Grawe BM, Kelly AM, Krych AJ, et al. Is grit the new fit?-assessing non-cognitive variables in orthopedic surgery trainees. J Surg Educ 2019; 76:924-30

19. Brateanu A, Switzer B, Scott SC, Ramsey J, Thomascik J, Nowacki AS, et al. Higher grit scores associated with less burnout in a cohort of internal medicine residents. Am J Med Sci 2020;360:357-62.

20. Sood A, Sharma V, Schroeder DR, Gorman B. Stress Management and Resiliency Training (SMART) program among Department of Radiology faculty: a pilot randomized clinical trial. Explore (NY) 2014;10:358-63.

21. Jung YJ, Park YY. The correlation between early childhood teacher of grit, emotional burnout, and job performance efficacy. Asia-Pac J Multimed Serv Converg Art, Humanit, Sociol 2019;9:313-22. 